\title{
Nanometers-thick self-organized Fe stripes: bridging the gap between surfaces and magnetic materials
}

\author{
O. Fruchart, ${ }^{1, \text { F }}$ M. Eleoui ${ }^{1}$ J. Vogel, ${ }^{1}$ P. O. Jubert, ${ }^{2}$ A. Locatelli, ${ }^{3}$ and A. Ballestrazzi ${ }^{3}$ \\ ${ }^{1}$ Laboratoire Louis Néel (CNRS, UPR5051), BP166, F-38042 Grenoble Cedex 9, France \\ ${ }^{2}$ IBM Research, Zürich Research Laboratory - Säumerstrasse 4, 8803 Rüschlikon, Switzerland \\ ${ }^{3}$ ELETTRA S.C.p.A., I-34012 Basovizza, Trieste, Italy
}

(Dated: November 20, 2018)

\begin{abstract}
We have fabricated $5 \mathrm{~nm}$-high $\mathrm{Fe}(110)$ stripes by self-organized (SO) growth on a slightly vicinal $\mathrm{R}(110) / \mathrm{Al}_{2} 0_{3}(11 \overline{2} 0)$ surface, with $\mathrm{R}=\mathrm{Mo}$, W. Remanence, coercivity and domain patterns were observed at room temperature (RT). This contrasts with conventional SO epitaxial systems, that are superparamagnetic or even non-magnetic at RT due to their flatness. Our process should help to overcome superparamagnetism without compromise on the lateral size if SO systems are ever to be used in applications.
\end{abstract}

Arrays of epitaxial nanometer-sized (1-50 nm) magnetic structures can be grown by self-organization ( $\mathrm{SO}$ ). However such structures are superparamagnetic or even non-magnetic at room temperature (RT) [1, 2, 3]. Indeed the energy barrier opposing spontaneous magnetization flipping roughly scales with $K V$, with $K$ the magnetic anisotropy per unit volume, and $V$ the system's volume. $3 \mathrm{D}$ clusters of similar lateral size can overcome superparamagnetism at RT by increasing $K[4]$. This seems not sufficient in epitaxial $\mathrm{SO}[\underline{3}, 5,6]$ because $\mathrm{SO}$ deposits are generally very flat, implying a very small $V$. Therefore, beating superparamagnetism in SO deposits without compromising on the lateral density seems to imply increasing their thickness $t$.

One way to force SO deposits to grow vertically and overcome superparamagnetism at RT is sequential deposition [7, 8]. We proposed a second route, that consists in annealing a thin continuous film deposited on a vicinal surface to form an array of several atomic layers (AL)-thick stripes [8, 9]. In the early reports, concerning $\mathrm{Fe} / \mathrm{Mo}(110)$ stripes, a stable thickness $t=6 \mathrm{AL}(\sim$ $1.2 \mathrm{~nm}$ ) was observed above 1-2 ALs of wetting. Yet this was not thick enough to observe static coercivity at RT, which could be obtained only for multidisperse assemblies of islands and stripes, thicker on the average. In this Letter we report the growth of thicker stripes, in the case of Fe/W(110): $t \sim 25 \mathrm{AL}(\sim 5 \mathrm{~nm})$. Such stripes display at RT functional features of magnetic materials: coercivity, remanence and domains, unlike conventional SO systems. The microscopic origin of the self-organization process is also unravelled.

The samples are epitaxially grown by pulsed laser deposition in a multi-chamber ultra-high vacuum setup (base pressure $7 \times 10^{-9} \mathrm{~Pa}$ ), with in situ STM, RHEED and Auger spectroscopy [10]. Commercial (11 $\overline{2} 0)$ sapphire wafers with a residual miscut angle $\epsilon<0.1^{\circ}$ are buffered with refractory metal films (Mo or W, $\lesssim 10 \mathrm{~nm}$-thick), whose surface consists of an array

*Olivier.Fruchart@grenoble.cnrs.fr of atomically-flat terraces of width $\sim 200 \mathrm{~nm}$, separated by mono-atomic steps [9]. Fe is then deposited at $150^{\circ} \mathrm{C}$ and annealed at $400-450^{\circ} \mathrm{C}$, covered with $1 \mathrm{~nm}$ Mo for controlling the magnetic anisotropy, and finally capped by $4 \mathrm{~nm} \mathrm{Al}$ as a protection against oxidation. AFM (PSI Autoprobe CP) and hysteresis loops (QD MPMS-XL) were performed ex situ. Samples were then dc-demagnetized ex situ with the field applied perpendicular to the stripes. Magnetic and chemical imaging was performed under zero external field using the Spectroscopic Photo Emission- and Low Energy Electron Microscope (SPELEEM), operational at the Nanospectroscopy beamline, at the Elettra synchrotron radiation facility in Trieste, Italy [11]. Element-selective magnetic contrast was obtained by combining energy filtered PEEM with Xray Magnetic circular dichroism (XMCD). The circularlypolarized X-ray beam was shone on the sample at an angle of incidence of $16^{\circ}$ and parallel to the in-plane [1 $\left.1 \overline{1} 0\right]$ of the sample, i.e. roughly perpendicular to the stripes. The photon energy was tuned to the $\mathrm{Fe}_{3}$ edge. The magnetic contrast $\left(I^{+}-I^{-}\right) /\left(I^{+}+I^{-}\right)$, or XMCD asymmetry, is proportional to the projection of the magnetization along the X-ray beam direction, where for each pixel $I^{+}$and $I^{-}$are the intensity acquired with opposite helicity of the photon beam. The chemical contrast is given by $\left(I^{+}+I^{-}\right) / 2$.

In conventional step decoration processes, the stripes have a height comparable to that of mono-atomic steps 2 , 3, 12. FIG. 11 reveals the microscopic mechanism allowing the array of mono-atomic steps to serve as a template for the self-organization of stripes much thicker than the steps themselves. The frontier between the low-temperature growth mode with increasing kinetic roughness [13] and the high-temperature StranskiKrastanov growth mode with dots formation 14 is $150{ }^{\circ} \mathrm{C}$. At this temperature the growth of $\mathrm{Fe}$ proceeds layer-by-layer on terraces, but grooves with a depth increasing with nominal Fe thickness are observed in register with the initial array of atomic steps (FIG. 10), probably resulting from stress effects [15] and the connection of interface dislocation arrays across steps 16. Annealing $\mathrm{Fe} / \mathrm{W}(110)$ films is known to yield stripes aligned 
along [001] 17. Here the grooves drive nucleation, yielding a self-organized array of stripes roughly aligned along

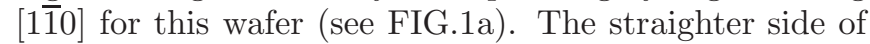
the stripes is the one lying along the steps of the buffer layer (FIG. 10-f). As grooves along buried steps have been observed in other systems [18], the self-organization process described here is expected to be of broader validity than solely for $\mathrm{Fe} / \mathrm{bcc}(110)$.

In the case of $\mathrm{Fe} / \mathrm{W}(110) / \mathrm{Al}_{2} \mathrm{O}_{3}(11 \overline{2} 0)$ (FIG. 2a, sample called $\mathrm{Fe} / \mathrm{W}$ in the following) the stripes display a sharp distribution function of height, centered around $t \sim 5.5 \mathrm{~nm}$ independent of nominal Fe thickness, a value that is much higher than for $\mathrm{Fe} / \mathrm{Mo}(110)(1.2 \mathrm{~nm}$, see above). For a second sample a $1 \mathrm{~nm}$-thick pseudomorphic W film was deposited onto a Mo(110) buffer layer; the absence of Mo segregation towards the surface was checked with a quantitative Auger analysis. For this composite buffer layer, Fe stripes with a monodisperse thickness $t \sim 4.3 \mathrm{~nm}$, again independent of nominal Fe thickness, are observed (FIG. 22, sample called Fe/W/Mo). This

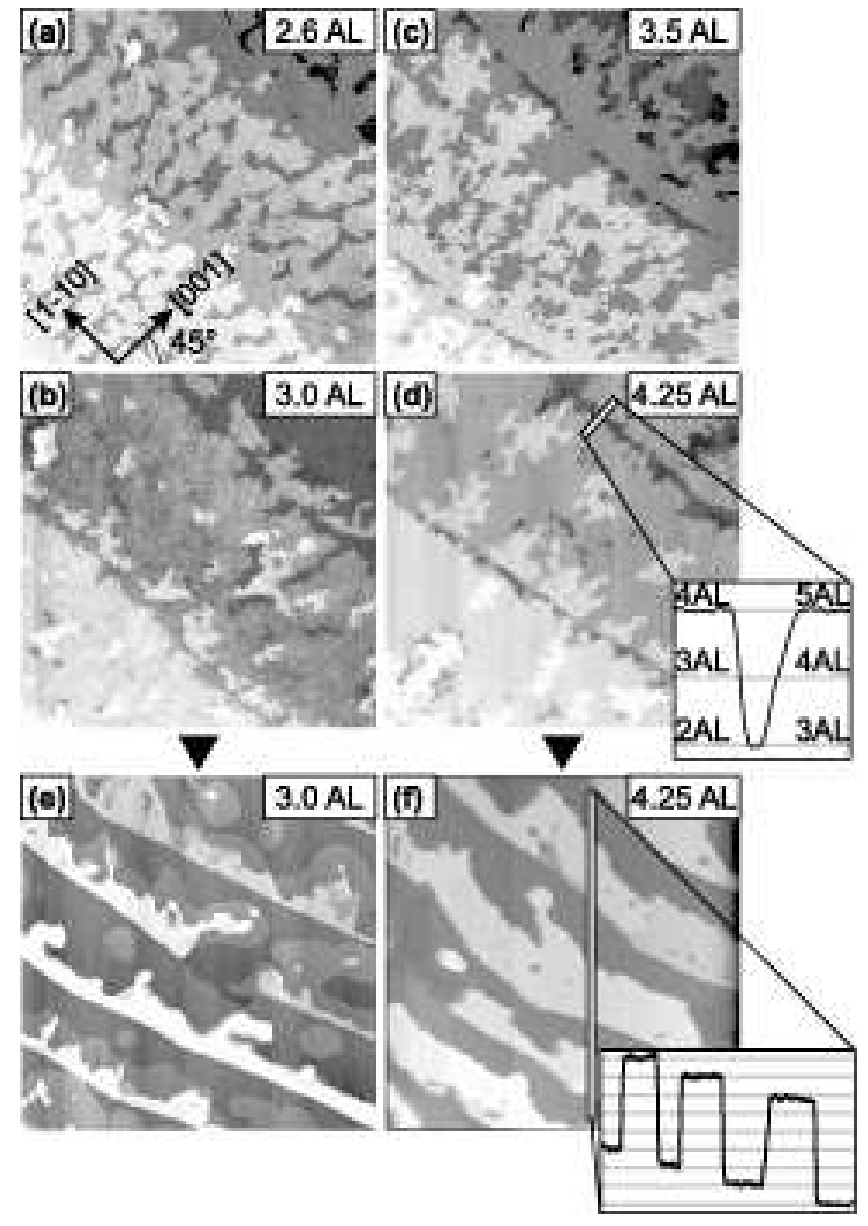

FIG. 1: STM pictures of $\mathrm{Fe} / \mathrm{Mo}(110)$ after growth at $150{ }^{\circ} \mathrm{C}(\mathrm{a}-\mathrm{d}, 400 \times 400 \mathrm{~nm})$ and after annealing at $450{ }^{\circ} \mathrm{C}(\mathrm{e}-\mathrm{f}$, $800 \times 800 \mathrm{~nm}$ ), for varying Fe nominal thickness (see upperright labels). The insets show cross-sections with integer ALs sketched by gray lines. suggests that both the lattice parameter of the buffer layer and the interfacial energy influence the stripe thickness. The origin of this metastable thickness is under investigation. For both samples the miscut angle is $0.04^{\circ}$, yielding a terrace width of $290 \mathrm{~nm}$. (a)

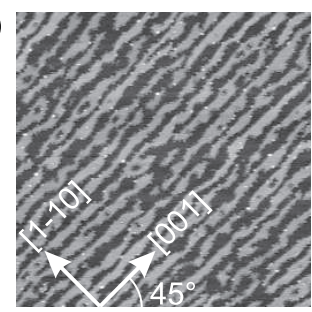

(c)

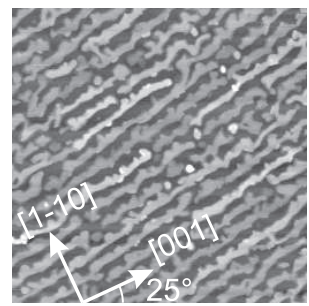

(b)

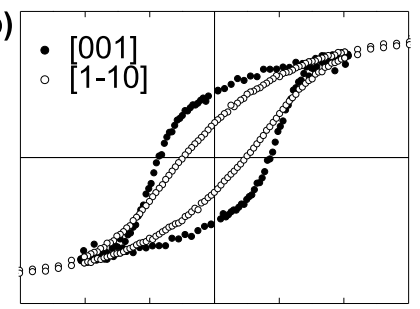

(d)

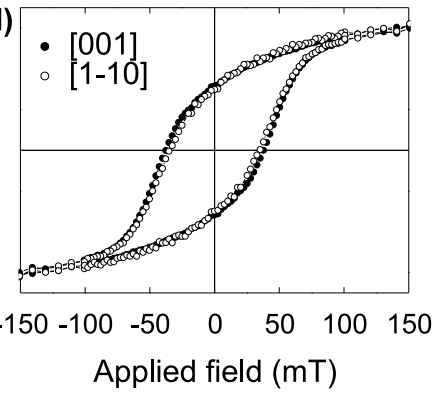

FIG. 2: $5 \times 5 \mu \mathrm{m}$ AFM pictures of samples (a) Fe/W and (c) Fe/W/Mo (see text for definition). The in-plane lattice directions are shown. In-plane hysteresis loops of samples (b) $\mathrm{Fe} / \mathrm{W}$ and (d) $\mathrm{Fe} / \mathrm{W} / \mathrm{Mo}$.

FIG. 2] shows RT hysteresis loops of sample Fe/W along two in-plane directions. [11̄0] is a hard magnetic axis and [001] is an easy axis, with significant remanence and a coercivity of $43 \mathrm{mT}$. The coercivity at $10 \mathrm{~K}$ is only slightly increased to $49 \mathrm{mT}$, confirming the weak effect of temperature that results from the large activation volume expected in thick stripes. This contrasts with the superparamagnetic behavior of conventional SO systems. FIG. 21 shows RT hysteresis loops of sample Fe/W/Mo. This time both in-plane axes are magnetically similar, with again significant remanence and a mean coercive field of $36 \mathrm{mT}$ (we do not discuss the origin of anisotropy in these samples, which is a complex balance between bulk, magneto-elastic, interface [19, 20, 21] and shape anisotropies).

FIG. 3 shows PEEM images of sample Fe/W/Mo, with a nominal thickness slightly smaller than in FIG. 22, yielding narrower and more irregular stripes (see chemical contrast on FIG. 3 ). In FIG. 3 3 ) light (resp. dark) areas correspond to magnetic domains pointing along [11̄0] (resp.[110]) (FIG. 2:), i.e. roughly perpendicular to the stripes. Grey areas correspond to domains along [001], i.e. roughly parallel to the stripes, or to the non-magnetic material between the stripes . These domain patterns are similar to those observed at the macroscopic scale 22]. They arise to satisfy both stray field flux-closure and charge-free domain walls. Depending on the local orientation of the stripes with respect to the crystallographic directions, $180^{\circ}$ and $90^{\circ}$ domain walls 
(a)

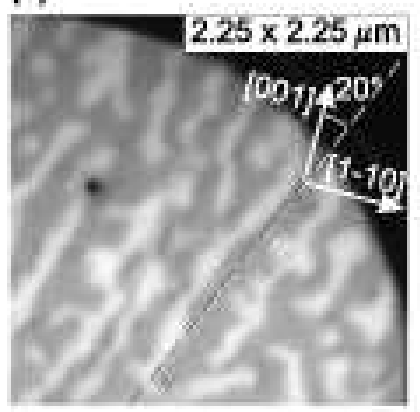

(b)
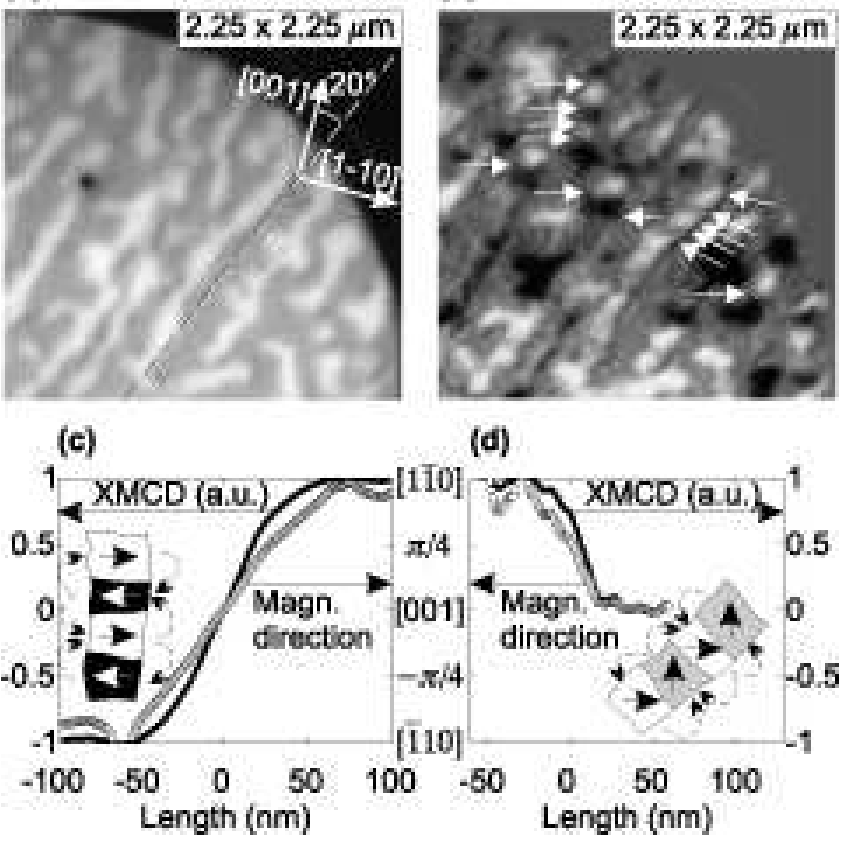

FIG. 3: (a) chemical and (b) magnetic $2.25 \times 2.25 \mu \mathrm{m}$ PEEM images of the same area of sample Fe/W/Mo. In (b) arrows pointing to the right (left) indicate some $180^{\circ}\left(90^{\circ}\right)$ domain walls. (c-d) profiles of $180^{\circ}$ (c) and $90^{\circ}$ (d) walls, obtained as the average of several cross-sections in (b) for (c), and in images with a total field of view of $2.5 \times 2.5 \mu \mathrm{m}$ (not shown here) for $90^{\circ}$ walls. The magnetization direction is calculated as $\arccos (\mathrm{XMCD})$. Insets: sketches of the flux closure domains indicated by a set of arrows in (b). The dotted-line arrows sketch the flux closure. are observed (FIG. 3 -d). We follow the usual definition of the width $\lambda$ of a wall as the width of the linear asymptote to the plot of magnetization angle versus length. We found (FIG. [3k-d) $\lambda_{180}=110 \mathrm{~nm}$ and $\lambda_{90}=50 \mathrm{~nm}$, respectively, satisfying $\lambda_{180} \sim 2 \lambda_{90}$ as expected. Note that the numerical value of $\lambda_{90}$ is however close to the expected lateral resolution of the microscope (a few tens of nm in PEEM mode).

To conclude we have unravelled a microscopic mechanism that allows a vicinal surface to serve as a template for the self-organized growth of stripes displaying a monodisperse thickness of up to $5.5 \mathrm{~nm}$. This growth process allows one to overcome superparamagnetism without compromising on the lateral density. Thus for the first time self-organized magnetic nanostructures were observed to display at room temperature usual features of bulk materials: remanence, coercivity and domain patterns.

\section{Acknowledgments}

We are grateful to $\mathrm{Ph}$. David and V. Santonacci for their technical support, and J. Camarero, F. Scheurer and Y. Samson for preliminary magnetic measurements. This work was partly funded by Région RhôneAlpes (Project Emergence 2001) and the French ministry of Research (ACI Nanostructures 2000).
[1] S. Padovani, I. Chado, F. Scheurer, and J.-P. Bucher, Phys. Rev. B. 59, 11887 (1999).

[2] J. Hauschild, U. Gradmann, and H. J. Elmers, Phys. Rev. B 57, R677 (1998).

[3] P. Gambardella, A. Dallmeyer, K. Maiti, M. C. Malagoli, W. Eberhardt, K. Kern, and C. Carbone, Nature 416, 301 (2002).

[4] S. Sun, C. B. Murray, D. Weller, L. Folks, and A. Moser, Science 287, 1989 (2000).

[5] P. Ohresser, N. B. Brookes, S. Padovani, F. Scheurer, and H. Bulou, Phys. Rev. B 64, 104429 (2001).

[6] P. Gambardella, S. Rusponi, M. Veronese, S. S. Dhesi, C. Grazioli, A. Dallmeyer, I. Cabria, R. Zeller, P. H. Dederichs, K. Kern, C. Carbone, and H. Brune, Science 300, 1130 (2003).

[7] O. Fruchart, M. Klaua, J. Barthel, and J. Kirschner, Phys. Rev. Lett. 83, 2769 (1999).

[8] O.Fruchart, P.-O. Jubert, C. Meyer, M. Klaua, J. Barthel, and J. Kirschner, J. Magn. Magn. Mater. 239, 224 (2002).

[9] P.-O. Jubert, O. Fruchart, and C. Meyer, J. Magn. Magn. Mater. 242-245, 565 (2002).

[10] P. O. Jubert, O. Fruchart, and C. Meyer, Surf. Sci. 522, 8 (2003).
[11] A. Locatelli, A. Bianco, D. Cocco, S. Cherifi, S. Heun, M. Marsi, M. Pasqualetto, and E. Bauer, J. Phys. IVproceedings 104, 99 (2003).

[12] J. Shen, M. Klaua, P. Ohresser, H. Jenniches, J. Barthel, C. V. Mohan, and J. Kirschner, Phys. Rev. B56, 11134 (1997).

[13] M. Albrecht, H. Fritzsche, and U. Gradmann, Surf. Sci. 294, 1 (1993).

[14] P.-O. Jubert, O. Fruchart, and C. Meyer, Phys. Rev. B 64, 115419 (2001).

[15] P. Müller, P. Turban, L. Lapena, and S. Andrieu, Surf. Sci. 488, 52 (2001).

[16] S. Murphy, D. Mac Mathúna, G. Mariotto, and I. V. Shvets, Phys. Rev. B 66, 195417 (2002).

[17] D. Sander, A. Enders, C. Schmidthals, D. Reuter, and J. Kirschner, Surf. Sci. 402-404, 351 (1998).

[18] S. Cherifi, C. Boeglin, S. Stanescu, J. P. Deville, C. Mocuta, H. Magnan, P. Le Fèvre, P. Ohresser, and N. B. Brookes, Phys. Rev. B 64, 184405 (2001).

[19] U. Gradmann, in Handbook of magnetic materials, edited by K. H. J. Buschow (Elsevier Science Publishers B. V., North Holland, 1993), vol. 7, chap. 1, pp. 1-96.

[20] R. M. Osgood III, R. L. White, and B. M. Clemens, Mat. Res. Soc. Symp. Proc. 384 (1995). 
[21] O. Fruchart, J.-P. Nozières, and D. Givord, J. Magn. Magn. Mater. 207, 158 (1999).

[22] A. Hubert and R. Schäfer, Magnetic domains. The analy- sis of magnetic microstructures (Springer, Berlin, 1999). 